\title{
Metalurgia Física
}

\section{Envelhecimento em um aço inoxidável AISI 304 contendo martensita induzida por deformação}

\author{
Cynthia Serra Batista Castro \\ Aluna de Pós-Doutorado do Departamento de Engenharia Metalúrgica e de Materiais da \\ Universidade Federal de Minas Gerais.E-mail: cynthiasbc@yahoo.com.br \\ Luciana Spíndola Sales \\ Aluna de Mestrado do Departamento de Engenharia Metalúrgica e de Materiais da \\ Universidade Federal de Minas Gerais. E-mail: luciana_spindola@yahoo.com.br \\ Berenice Mendonça Gonzalez \\ Professora Adjunta do Departamento de Engenharia Metalúrgica e de Materiais da \\ Universidade Federal de Minas Gerais. E-mail: gonzalez@demet.ufmg.br
}

\section{Resumo}

O envelhecimento na martensita no aço inoxidável austenítico AISI 304 pré-deformado em tração de 25\% a $5^{\circ} \mathrm{C}$ foi estudado com o objetivo de se determinarem os aspectos fenomenológicos e os mecanismos relacionados ao envelhecimento. As condições de pré-deformação utilizadas, de $25 \%$ a $-5^{\circ} \mathrm{C}$, resultaram em uma fração volumétrica de martensita igual a 60\%. Os tratamentos de envelhecimento foram realizados na faixa de temperatura de 80 a $160^{\circ} \mathrm{C}$, em intervalos de tempo variando de 1 a 400 minutos. Observou-se que o limite de escoamento aumenta com o aumento do tempo e da temperatura de envelhecimento,saturando em torno de 20 minutos a $160^{\circ} \mathrm{C}$. Encontrou-se um valor igual a $1 / 2$ para o expoente do tempo na equação que descreve a lei cinética do envelhecimento. Esse valor foi associado à formação de precipitado $\varepsilon$. O valor da energia de ativação obtido, $65 \mathrm{~kJ} / \mathrm{mol}$, sugere que o processo de envelhecimento é controlado pela difusão do carbono na martensita.

Palavras-chave: Martensita induzida por deformação, envelhecimento após deformação, carboneto $\varepsilon$.

\begin{abstract}
Strain aging of martensite in austenitic stainless steel AISI 304, 25\% pre-deformed at $-5^{\circ} \mathrm{C}$, was studied to determine phenomenological aspects and mechanisms of strain aging. Under these conditions, the result was a martensite volume fraction of $60 \%$. Ageing treatments were performed from 80 to $160^{\circ} \mathrm{C}$ during 1 to 400 minutes. Yield strength increases with the increase of aging time and temperature, saturation being reached in about 20 minutes at $160^{\circ} \mathrm{C}$. A time exponent of $1 / 2$ was found for the equation that describes the kinetics of the process. This value was associated to the formation of e precipitates. The value of the activation energy $(65 \mathrm{~kJ} / \mathrm{mol})$ was related to the diffusion of carbon in martensite.
\end{abstract}

Keywords: Strain induced martensite, strain ageing, $\varepsilon$ carbide. 


\section{Introdução}

O controle do envelhecimento, após deformação e envelhecimento dinâmico, no processamento termomecânico de aços, tem se destacado, cada vez mais, devido às importantes conseqüências práticas que determinam a aptidão à conformação ou o comportamento em serviço dessa classe de materiais. Os estudos, nessa área, visam à otimização do potencial de aumento de resistência associado ao envelhecimento, como, por exemplo, os aços para a indústria automobilística com envelhecimento controlado,"bake hardening” (De et al., 2000, Zhao et al., 2001, Souza e Buono, 2003; Bernebi et al., 2004), ou a minimização dos efeitos desse processo que comprometem o desempenho nas operações de conformação de produtos obtidos a partir de fio máquina de aço de baixo ou alto teor de carbono, como aços para parafusos e "steel cord” (Weidig et al., 1995; Hammerle et al., 2004; Castro et al., 1999 ; Buono et al., 1998; Gonzalez et al., 2003). Também, no caso de aços estruturais, o envelhecimento por deformação tem um efeito determinante em certas propriedades, como é o caso da resistência ao fogo (Santos, 2004).

Em aços inoxidáveis metaestáveis, como o aço AISI 304, a transformação da austenita em martensita induzida por deformação aumenta a aptidão à estampagem por acarretar um acréscimo na capacidade de encruamento para maiores valores de deformação e, portanto, um aumento na deformação uniforme. No entanto, a ação combinada de aumento de temperatura e deformação, durante a operação de estampagem, pode levar ao envelhecimento por deformação da martensita, o que prejudica o desempenho do material na prensa (Rathbun et al., 2000).

O número de trabalhos considerando o envelhecimento por deformação em aços inoxidáveis contendo martensita é pequeno, sendo que, para o aço AISI 304, não há referências na literatura sobre esse fenômeno. Rathbun et al. (2000) estudaram o envelhecimento na martensita induzida por deformação em aços inoxidáveis austeníticos AISI 301 e AISI 305. Os valores de energia de ativação encontrados se situaram entre 7 e $14 \mathrm{~kJ} /$ mol, o que levou os autores a associar o mecanismo de envelhecimento a uma redistribuição de curto alcance dos átomos de $\mathrm{C}$ e $\mathrm{N}$ nas deslocações.

Talonen et al. (2004) estudaram esse fenômeno em aços inoxidáveis austeníticos AISI 301 para diferentes pré-deformações, porém as energias de ativação encontradas por esses autores, 130, 134 e $113 \mathrm{~kJ} / \mathrm{mol}$, indicam que o processo foi avaliado na fase austenítica e não na martensita induzida por deformação, como haviam discutido. Esses autores relatam que o expoente do tempo, n, variou de acordo com a quantidade de prédeformação e temperatura de envelhecimento. Eles afirmaram que nenhum estudo relacionado à cinética de envelhecimento tinha sido proposto na literatura e que os valores encontrados para o expoente do tempo sugerem trabalhos posteriores. Sendo assim, o estudo do envelhecimento em aços inoxidáveis austeníticos do tipo AISI 304 é de grande interesse.

Esse trabalho envolve o estudo de um aço do tipo AISI 304, visando a estabelecer a influência do envelhecimento após deformação na estampagem de aços inoxidáveis austeníticos contendo martensita induzida por deformação, através da determinação da cinética de envelhecimento e da identificação dos mecanismos responsáveis pelo processo de envelhecimento na martensita nesse aço.

\section{Materiais e métodos}

Estudou-se um aço inoxidável austenítico de procedência comercial, do tipo AISI 304, processado industrialmente e recebido na forma de chapas recozidas de $0,6 \mathrm{~mm}$ de espessura, cuja composição química está apresentada na Tabela 1.

Amostras desse aço foram pré-deformadas em tração em temperatura infe-

Tabela 1 - Composição química do aço estudado (\% em peso).

\begin{tabular}{c|c|c|c|c|c|c}
\hline $\mathbf{C}$ (ppm) & Si & Mn & Cr & Ni & Cu & N (ppm) \\
\hline 255 & 0,44 & 1,13 & 18,23 & 8,91 & 0,23 & 474 \\
\hline
\end{tabular}
transformar parte da austenita em martensita induzida por deformação. Amostras retiradas, na direção de laminação das chapas, com comprimento útil de $80 \mathrm{~mm}$, largura de $12,5 \mathrm{~mm}$ e $0,6 \mathrm{~mm}$ de espessura, conforme especificado na norma ASTM E8M-98, para ensaio de tração em corpos de prova planos, foram utilizadas para caracterização estrutural e mecânica. Nos ensaios mecânicos, a taxa de deformação foi de $2,0 \times 10^{-3} \mathrm{~s}^{-1}$ e as propriedades mecânicas determinadas foram limite de escoamento, $\sigma_{\mathrm{e}}$, limite de resistência, $\sigma_{R}$, alongamento uniforme, $\mathrm{e}_{\mathrm{u}}$, e alongamento total, $\mathrm{e}_{\mathrm{T}}$.

Os corpos de prova foram pré-deformados de $25 \%$ a $-5^{\circ} \mathrm{C}$ em tração em uma máquina universal de ensaios marca Instron, modelo 5582, equipada com câmara para testes, entre $-30^{\circ} \mathrm{C}$ e $200^{\circ} \mathrm{C}$, e pacotes de programas para aquisição e tratamento de dados (Instron, Series IX para Windows), cujos valores foram utilizados como base para o cálculo da variação das propriedades mecânicas com o tempo e temperatura de envelhecimento. Os tratamentos térmicos de envelhecimento foram realizados nas amostras pré-deformadas nas condições descritas anteriormente, em um banho de óleo de silicone equipado com controlador de temperatura e bomba de agitação mecânica. A capacidade do banho (8 litros de óleo), aliada à agitação mecânica, proporciona variações de temperatura menores de $\pm 1^{\circ} \mathrm{C}$ e tempos de equalização inferiores a 50 segundos. Os tempos de envelhecimentos foram contados a partir da equalização da temperatura. Foram consideradas temperaturas de envelhecimento entre $80^{\circ} \mathrm{C}$ e $160^{\circ} \mathrm{C}$ e tempos de envelhecimento entre 1 e 400 minutos. Após os tratamentos no banho de óleo de silicone, as amostras foram submetidas ao ensaio de tração até a ruptura do corpo de prova. rior à temperatura ambiente, de forma a 
A fração volumétrica de martensita foi determinada em amostras pré-deformadas de $25 \%$ a $-5^{\circ} \mathrm{C}$ e envelhecidas por 60 minutos nas diferentes temperaturas com o objetivo de estabelecer o efeito do tratamento de envelhecimento na fração volumétrica de martensita. A medida da fração volumétrica de martensita foi feita por difração de raios X, em um difratômetro Philips PW1710, utilizando radiação $\mathrm{Cu}-\mathrm{K} \alpha$ e um cristal monocromador de grafita. Amostras medindo 20mm x 20mm foram seccionadas em cortadeira metalográfica. Para eliminar a superfície afetada pelo corte, onde poderia haver martensita induzida por deformação, as amostras foram decapadas em uma solução contendo $\mathrm{HCl}$, ácido acético e $\mathrm{HNO}_{3}$, que foi escolhida com base em vários testes preliminares realizados por Castro (2003). As quantidades relativas de fases foram, também, determinadas, utilizando a difração de raios X.

A determinação da susceptibilidade ao envelhecimento dos aços foi feita a partir das medidas das intensidades da variação do limite de escoamento, nas condições de tempo e temperatura de envelhecimento em que essas variações foram máximas.

A cinética de envelhecimento foi determinada através da equação de Harper (Leslie, 1982), conforme as equações:

$$
-\ln (1-w)=\left(\frac{t}{\tau}\right)^{n}=(k t)^{n}
$$

onde: $\tau$ e $n$ são constantes do tempo, $w$ é a fração transformada e $k$ é constante da velocidade onde:

$$
k=k_{0} \exp \left(\frac{-Q}{R T}\right)
$$

Nessa equação $k_{0}$ é uma constante, $Q$ é a energia de ativação do processo, $R$ é a constante universal dos gases e $T$ é a temperatura absoluta. Nas equações (1) e (2), os valores de $n$ e $Q$ dependem do mecanismo responsável pelo processo de envelhecimento.

Como o aumento do limite de escoamento é causado pela segregação de átomos intersticiais para as deslocações, então:

$W=\frac{\Delta \sigma_{(t)}}{\Delta \sigma_{\text {máx }}}$

onde $\Delta \sigma_{\mathrm{t} \text { ? }}$ é o aumento no limite de escoamento após o tempo t e temperatura $\mathrm{T}$ de envelhecimento e $\Delta \sigma_{\text {máx. }}$ é o valor de saturação do limite de escoamento.

A partir dos aspectos fenomenológicos observados e dos valores obtidos para n e Q, foram identificados os mecanismos envolvidos no envelhecimento da martensita induzida por deformação no aço inoxidável austenítico AISI 304.

\section{Resultados e discussão}

As propriedades mecânicas do aço inoxidável austenítico, como recebido e pré-deformado em tração de $25 \%$ a $-5^{\circ} \mathrm{C}$, estão apresentadas na Tabela 2, na qual pode-se observar uma grande variação nas propriedades mecânicas do aço após a formação de martensita induzida por deformação. As propriedades mecânicas foram avaliadas em termos do limite de escoamento, $\sigma_{\mathrm{e}}$, limite de resistência, $\sigma_{\mathrm{R}}$, alongamento uniforme, $\mathrm{e}_{\mathrm{u}}$, e alongamento total, $\mathrm{e}_{\mathrm{T}}$.

A Figura 1 mostra as curvas tensão-deformação nominais para o material como recebido e pré-deformado. No caso do material como recebido observa-se uma curva $\sigma x e$ característica de um aço inoxidável austenítico recozido: ausência de limite de escoamento definido e elevadas capacidade de encruamento e deformação uniforme (Pickering, 1976). No que se refere ao material pré-deformado, além do aumento na resistência e decréscimo no valor do alongamento, observam-se, também, a presença de limite de escoamento definido e um decréscimo na capacidade de encruamento, característicos da influência da presença de martensita induzida por deformação no comportamento mecânico do aço.

Para avaliar se as mudanças observadas nas propriedades mecânicas estariam associadas ao envelhecimento na martensita induzida por deformação, uma amostra do aço 100\% austenítico foi envelhecida a $150^{\circ} \mathrm{C}$ durante 200 minutos. Como se pode observar na Figura 2, não há, praticamente, nenhuma mudança nas propriedades mecânicas do aço inoxidável austenítico AISI 304 envelhecido, quando comparado ao aço como recebido, indicando que as mudanças nas propriedades mecânicas, observadas após pré-deformação em tração e posterior envelhecimento, estão associadas à martensita induzida por deformação.

Os aumentos observados nas propriedades mecânicas, para o aço estudado, variam com o tempo e a temperatura de envelhecimento, conforme se pode observar na Figura 3. As curvas de variação do limite de escoamento foram obtidas para o aço tratado entre 80 e $160^{\circ} \mathrm{C}$. Os pontos da curva foram determinados a partir da média de três ensaios. Observa-se, nessa figura, que há um aumento contínuo do limite de escoamento em função do tempo e temperatura de envelhecimento. $\mathrm{O}$ valor de saturação dessa propriedade acontece em torno de 20 minutos, a $160^{\circ} \mathrm{C}$.

Os difratogramas de raios $\mathrm{X}$ do aço AISI 304 são mostrados na Figura 4 (a) e (b). A Figura 4 (a) mostra o difratograma do aço como recebido, sendo a austenita a única fase presente, como era de se esperar. A Figura4 (b) mostra o difratograma da amostra pré-deformada de $25 \%$ a $-5^{\circ} \mathrm{C}$ em tração.

Tabela 2 - Propriedades mecânicas do aço AISI 304 como recebido (CR) e pré-deformado (PD).

\begin{tabular}{c|c|c|c|c}
\hline Aço & $\sigma_{e}(\mathrm{MPa})$ & $\sigma_{\mathrm{R}}(\mathrm{MPa})$ & $\mathbf{e}_{\mathrm{u}}(\%)$ & $\mathbf{e}_{\mathrm{T}}(\%)$ \\
\hline $\mathbf{C R}$ & $260 \pm 10$ & $643 \pm 13$ & $56,82 \pm 9,0$ & $60,44 \pm 5,3$ \\
\hline $\mathbf{P D}$ & $940 \pm 12$ & $1004 \pm 26$ & $13,04 \pm 1,5$ & $15,52 \pm 2,3$ \\
\hline
\end{tabular}




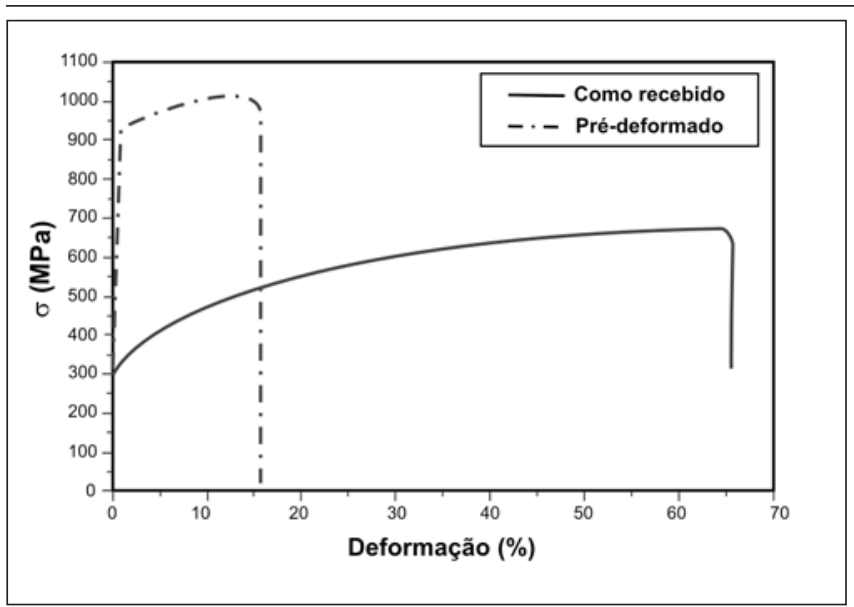

Figura 1 - Ensaio de tração do aço AISI 304 como recebido e pré-deformado de $25 \%$ a $-5^{\circ} \mathrm{C}$.

Conforme pode-se observar nesse difratograma, a intensidade dos picos da austenita diminuiu, quando comparada à Figura 4 (a), enquanto a intensidade dos picos da martensita $\alpha$ ' aumentou. Nessa figura, não se observa a presença da martensita $\varepsilon$, o que está de acordo com os trabalhos de Cina (1958, 1954) e Spencer et al. (2004), que relatam que a martensita $\varepsilon$ está presente somente em pequenas deformações e diminui para altos valores de deformação. Segundo os autores, a martensita $\alpha$ ' é nucleada a partir da martensita $\varepsilon$.

O cálculo da fração envelhecida, em termos da variação das propriedades mecânicas desse material, foi realizado levando-se em consideração a variação do limite de escoamento inferior, $\sigma_{\mathrm{e}}$. O cálculo da fração envelhecida, em termos da variação do limite de escoamento no envelhecimento, pode ser feito através da relação apresentada na equação 3, onde:

$y=w=\frac{\Delta \sigma_{t}}{\Delta \sigma_{\text {máx. }}}=\frac{\sigma_{t}-\sigma_{0}}{\sigma_{\text {máx. }}-\sigma_{0}}$

$\sigma_{t}$ é o limite de escoamento, medido após o tratamento de envelhecimento por um tempo $t$ a uma dada temperatura de envelhecimento, $\sigma_{0}$ é o limite de escoamento da amostra pré-deformada e $\sigma_{\text {máx }}$ o o valor de saturação de $\sigma_{\mathrm{e}}$.

Calculando-se os valores de $y$ através da equação 4 , determinam-se os parâmetros que caracterizam a cinética de envelhecimento, que são o expoente do tempo, $n$, e a constante de velocidade da reação, $k$, através da linearização da equação 1 :

$\ln \ln \left[\frac{1}{(1-y)}\right]=n \ln \mathrm{k}+\mathrm{n} \ln \mathrm{t}$

Assim, o expoente do tempo, $n$, é dado pela inclinação das curvas $\ln \ln \left[\frac{1}{(1-y)}\right]$ versus $\operatorname{lnt}$. O valor de lnk é obtido a partir do intercepto da reta.

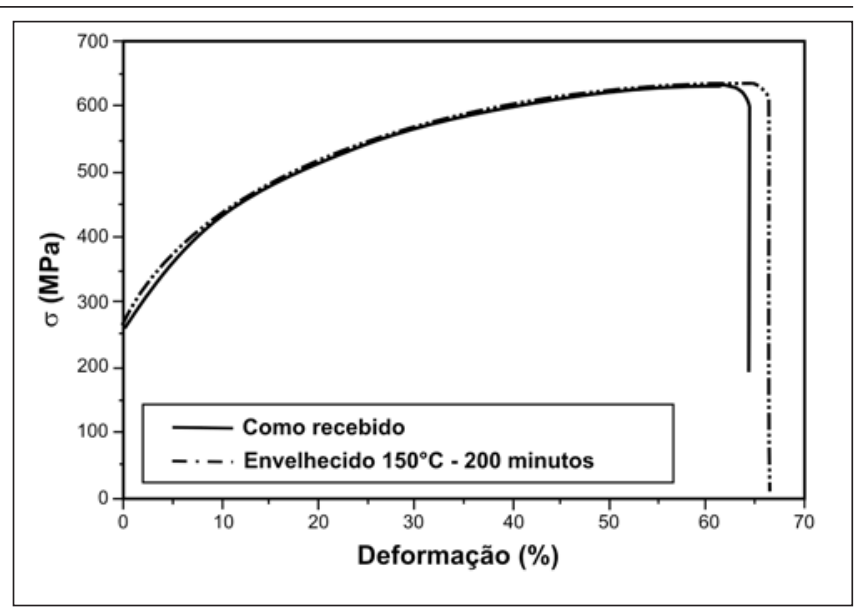

Figura 2 - Efeito do envelhecimento a $150^{\circ} \mathrm{C}$ e 200 minutos nas propriedades mecânicas do aço AISI 304.

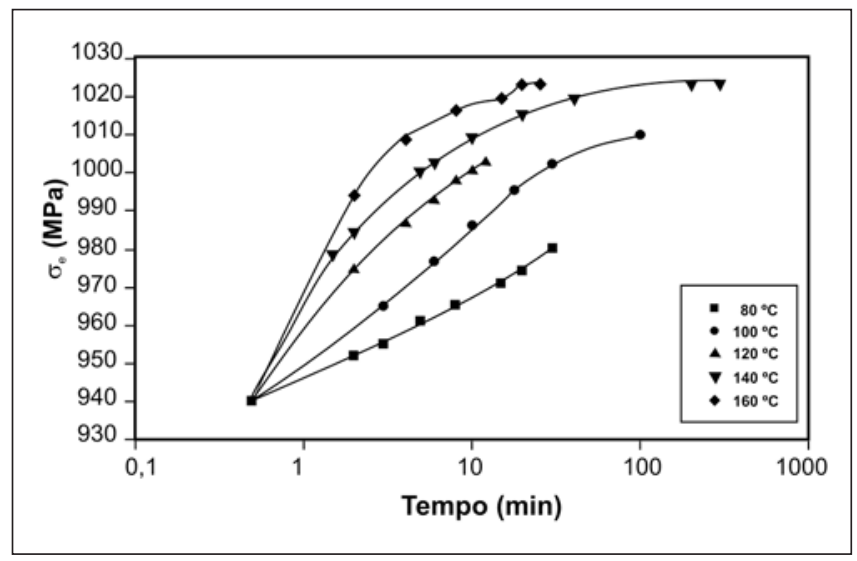

Figura 3 - Variação do limite de escoamento, $\sigma_{\mathrm{e}}$, com o tempo de envelhecimento, no aço AISI 304 pré-deformado de $25 \%$ a $-5^{\circ} \mathrm{C}$ em tração e envelhecido nas condições indicadas.

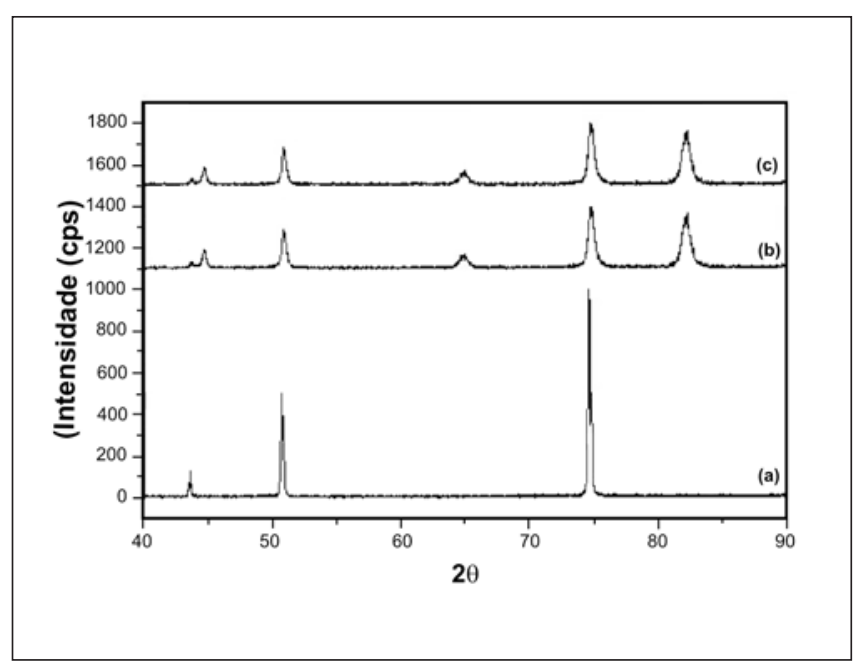

Figura 4 - Difratograma de raios X do aço AISI 304 (a) como recebido e (b) Pré-deformado em tração de $25 \%$ a $-5^{\circ} \mathrm{C}$. 
A Figura 5 mostra a linearização da fração envelhecida referente ao aço estudado, avalianddo-se $\sigma_{\mathrm{e}}$.

O expoente do tempo obtido, nas temperaturas estudadas, variou de 0,47 a 0,5 , podendo ser considerado igual a $1 \frac{1}{2}, 0$ que está de acordo com a lei cinética para precipitação do carboneto $\varepsilon$ na martensita (Waterschoot et al., 2003; De et al., 2001).

$\mathrm{O}$ valor de $\mathrm{Q}$ foi obtido pela inclinação da reta que descreve a variação de lnk em função de $1 / T$, -Q/R. O valor obtido, $65 \mathrm{~kJ} / \mathrm{mol}$, é próximo ao valor de $70 \mathrm{~kJ} / \mathrm{mol}$ determinado por De et al. (2001) para a precipitação de carboneto $\varepsilon$. Esse valor é da mesma ordem de grandeza do valor da energia de ativação para difusão do carbono na ferrita $\alpha$, o que poderia ser esperado, uma vez que a ferrita $\alpha$ e a martensita $\alpha$ ' apresentam mesma estrutura cristalina (CCC) e o processo é controlado pela difusão do carbono na fase martensítica.

\section{Conclusões}

Os valores do expoente do tempo e da energia de ativação determinados para o envelhecimento, na martensita induzida por deformação no aço AISI 304, indicam que as variações nas propriedades mecânicas decorrentes do envelhecimento são devidas à precipitação do carboneto $\varepsilon$ na martensita.

\section{Agradecimentos}

Os autores agradecem à CAPES e à FAPEMIG pelo apoio financeiro.

\section{Referências Bibliográficas}

BERNEBI, S., FAVIER, V., LEMOINE, X., BERVEILLER, M. A micromechanical approch to model to bake hardening effect for low carbon steels, Scripta Materialia, v.51, n2, 2004, p.303.

BUONO, V. T. L., ANDRADE, M. S. AND GONZALEZ, B. M. Kinectics of strain aging in drawn pearlitic steels, Metalurgical and Materials Transactions A, v. 29, n. 5, p.1415-1423, 1998.

CASTRO, C. S. B., BUONO, V. T. L., ANDRADE, M. S., PAULA E SILVA, E. M., GONZALEZ, B. M. Strain aging of pearlitic wire rod, Wire Journal International, v.32, n.11, p.108-112, 1999.

CASTRO, C. S. B. Efeito da adição de cobre nas propriedades mecânicas de aços inoxidáveis austeníticos. CPGEM, UFMG, 2003. 182p. (Tese de Doutorado).

CINA, B. Effect of cold work on the $\gamma \rightarrow \alpha$ ' transformation in some Fe-Ni-Cr alloys. Journal of the Iron and Steel Institute, v.177, n.8, p.406-422, 1954.

CINA, B. A transitional hcp phase in the $\gamma \rightarrow \alpha$ ' transformation in certain Fe-base alloys. Acta Metallurgica, v. 6, n. 12, p.748-762, 1958.

DE, A. K., VANDEPUTTE. S., DE COOMAN, B. C. Kinetics of low temperature precipitation in a ULC - Bake Hardening Steel. Scripta Materialia, v. 44, 2001, p. 695-700.

GONZALEZ, B. M., BUONO, V. T. L., MARCHI, L. A.,

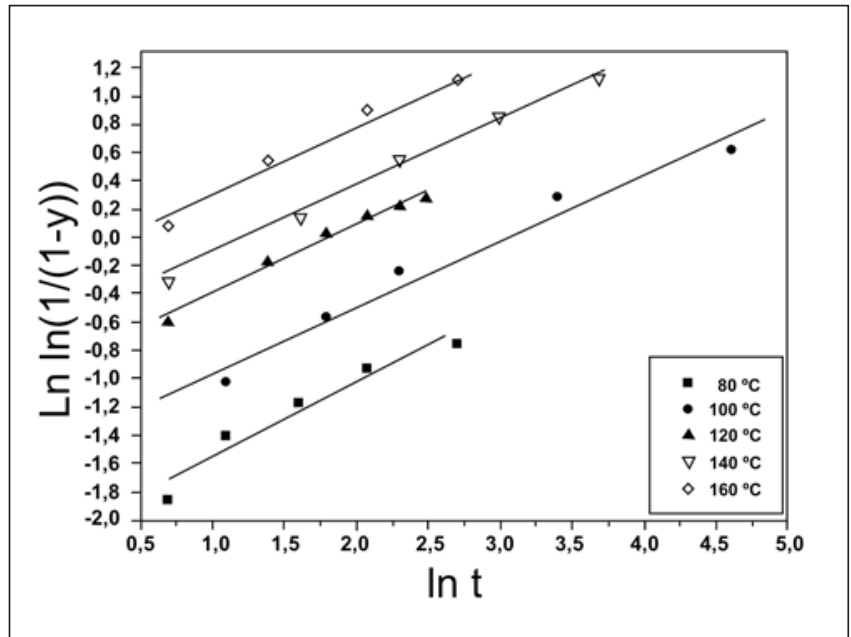

Figura 5 - Linearização da fração envelhecida, y, com o tempo de envelhecimento conforme equação 5 .

FONSECA, E. J., MODENESI, P. J. Measurements of dynamic strain aging in pearlitic steels by tensile tests, ISIJ Interational, v.43, n3, p.428-432, 2003.

HAMMERLE, J. R., ALMEIDA, L. H., MONTEIRO, S. N. Lower temperature mechanism of strain aging in carbon steels for drawn wires, Scripta Materialia, v.50, p.1289, 2004.

LESLIE, W. C. The physical metallurgy of steels. New York: McGrawHill, 1982. 396p.

PICKERING, F. B. Physical metallurgy of stainless steel. International Metals Reviews, rev. 211, p. 227-268, 1976.

RATHBUN, R. W., MATLOCK, D. K., SPEER, J. G. Strain aging behaviour of austenitic stainless steels containing strain induced martensite. Scripta Materialia, v.42, p.887-891, 2000.

SANTOS, O. J. Caracterização estrutural e mecânica de aços resistentes ao fogo a base de Mo-Si-P. CPGEM, UFMG, 2004. 135p. (Tese de Doutorado).

SOUZA, T. O., BUONO, V. T. L. Optimization of the strain aging resistence in aluminum killed steels produced by continuous annealing, Materials Science and Engineering A, v.354, n.1-2, p.212-216, 2003.

SPENCER, K., EMBURY, J. D., CONLON, K. T., VÉRON, M., BRÉCHET, Y. Strengthening via the formation of strain-induced martensite in stainless steels. Materials Science and Engineering $\boldsymbol{A}$, article in press, jul. 2004.

TALONEN, J., NENONEN, P., HÄNNINEN, H. Static Strain ageing of cold-worked austenitic stainless steel. In: HIGH NITROGEN STEELS - INTERNATIONAL CONFERENCE, 2004, Ostend. Proceedings... Ostend: Grips Media, 2004. p.113-122.

WATERSCHOOT, T., DE, A. K., VANDEPUTTE, S., DE COOMAN, B. C. Static Strain Aging Phenomena in Cold-Rolled Dual-Phase Steels. Metallurgical and Materials Transactions A, v. 34A, p. 781-791, 2003.

WEIDIG, C., ESPÍDOLA, M. M., GONZALEZ, B. M., RODRIGUES P. C. M., ANDRADE, M. S. Dynamic strain aging in low carbon steel wire rods, Wire Journal International, v.28, n1, 1995, p.82-85.

ZHAO, J. Z., DE, A. K., DE COOMAN, B. C. Formation of Cottrell atmosphere during strain aging of bake-hardenable steels, Metalurgical and Materials Transactions A, v.32, 2001, p.417.

Artigo recebido em 30/07/2006 e aprovado em 05/10/2006. 